Vol. 6(5), pp. 68-74, September 2014

DOI: $10.5897 / J E T R 2014.0383$

Article Number: CBCA8AD47531

ISSN 2006-9790

Journal of Engineering and Technology

Copyright (C) 2014

Research

Author(s) retain the copyright of this article

http://www.academicjournals.org/JETR

\title{
Effect of immersion speed on the mechanical properties and micro-structure of oil quenched AISI 1020 steel
}

\author{
Mudasiru L. O. ${ }^{1 \star}$, Babatunde I. A. ${ }^{1}$, Raheem W. A. ${ }^{1}$ and Lasisi A. K. ${ }^{2}$ \\ ${ }^{1}$ Department of Mechanical Engineering, Ladoke Akintola University of Technology, Ogbomosho, Nigeria. \\ ${ }^{2}$ Department of Mechanical Engineering, Kwara State Polytechnic, Ilorin, Kwara State, Nigeria.
}

Received 3 July, 2014; Accepted 27 August, 2014.

\begin{abstract}
Steel is one of the most important commodities in the engineering world due to its usefulness and availability. Its application is wider than any other metal, ranging from domestic use in the forms of utensils and many more to its use in construction and manufacturing industries. The wide range of application of steel in engineering practices has prompted the desire to increase certain mechanical properties to meet engineering requirements through heat treatment. In this work, samples of the specimen were purchased at a local market and the spectrometry analysis was carried out. The steel samples were machined into required shape, heat-treated and cooled under different immersion speeds. The effects of immersion speeds on the mechanical properties (hardness, \% elongation, tensile strength, modulus of elasticity and yield strength) and microstructure of the quenched AISI 1020 were investigated. The result obtained revealed an improvement in the mechanical properties of the materials at different immersion speed.
\end{abstract}

Key words: Heat treatment, mechanical properties, microstructure, AISI 1020.

\section{INTRODUCTION}

Carbon steel is a type of steel where the main interstitial alloying constituent is Carbon in the range of 0.12 to 2.0\% (Çalik, 2009; Sharma et al., 2012; Fadare, 2011). The American Iron and Steel Institute (AISI) defines Carbon steel as a steel in which no minimum content is specified or required for Chromium, Cobalt, Molybdenum, Nickel, Niobium, Titanium, Tungsten, Vanadium, Zirconium, or any other element added to obtain a desired alloying effect. According to Odusote et al. (2012), steels with Carbon content varying from 0.25 to $0.65 \%$ are classified as medium Carbon, while those with Carbon content less than $0.25 \%$ are termed low Carbon. The Carbon content of high Carbon steels usually ranges within 0.65 to $1.5 \%$.

Generally, the mechanical properties of plain Carbon

*Corresponding author. E-mail: owolatif2000@gmail.com

Author(s) agree that this article remain permanently open access under the terms of the Creative Commons Attribution License 4.0 International License 
Table 1. Composition of the low-Carbon steel.

\begin{tabular}{cccccccc}
\hline $\mathbf{C ~}(\%)$ & $\mathbf{S i}(\%)$ & $\mathbf{M n}(\%)$ & $\mathbf{S ~ ( \% )}$ & $\mathbf{P ~ ( \% )}$ & $\mathbf{C r}(\%)$ & $\mathbf{N i}(\%)$ & $\mathbf{C u}(\%)$ \\
\hline 0.189 & 0.207 & 0.497 & 0.021 & 0.022 & 0.101 & 0.073 & 0.174 \\
\hline
\end{tabular}

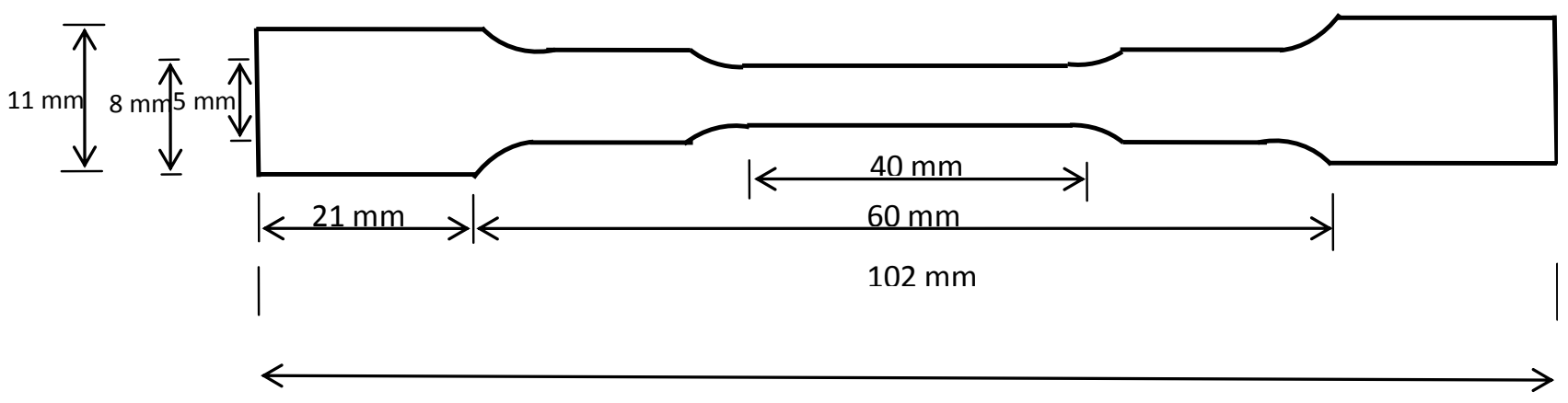

Figure 1. Machined specimen for tensile test.

steels increases with the increased Carbon concentration dissolved in austenite prior to quenching during hardening heat treatment, which may be due to transformation of austenite into martensite (Odusote et al., 2012). Therefore, the mechanical strength of medium Carbon steels can be improved by quenching in appropriate medium. The major influencing factors in the choice of the quenching medium are the kind of heat treatment to be employed, composition of the steel, the sizes and shapes of the parts (Odusote et al., 2012).

Quenching is a common manufacturing process aiming to produce components with desirable properties such as low residual stresses and distortions, avoidance of cracks, specific hardness, and achievement of improved properties (Mackerle, 2003). Low Carbon steel which was employed in the course of this study has high malleability and ductility, good machinability, good surface finish, good formability and general availability.

\section{MATERIALS AND METHODS}

Samples of steel bar purchased from a local market in Oyo State, South-Western Nigeria, were cut into $25 \mathrm{~mm}$ diameter and $100 \mathrm{~mm}$ long. Also, the determination of the chemical composition of the specimen was carried out using Minipal 4 Spectrometer (Table 1).

\section{Specimen preparation}

Each specimen was prepared for hardness and tensile tests. The samples were machined into standard specifications for tensile testing using Monsanto Tensiometer, following standard test procedures in accordance with the ASTM E8M-91 standards
(1992) (Figure 1). An optical metallurgical microscope XJL-17 model with magnification $10 \times 10$ was used for taken the micrograph of the samples.

\section{Heating and quenching process}

Heating of the specimen was carried out at the metrology laboratory of the Mechanical Engineering Department, Ladoke Akintola University of Technology, Nigeria. This process involved heating the tensile specimen to an austenising temperature of $850^{\circ} \mathrm{C}$. The material was maintained at this temperature for $1 \mathrm{~h}$ and then quenched at various immersion speeds of $0.3102,0.3241,0.3384$, and $0.4167 \mathrm{~m} / \mathrm{s}$ respectively, using oil as the quenchant.

\section{Mechanical tests}

After quenching operation, the materials were returned to the Engineering Materials Development Institute (EMDI) Akure, where the effects of the quenching at different immersion speeds on the physical and microstructural properties of the steel samples were determined.

\section{Microstructure analysis}

The microstructural analysis was obtained using an optical metallurgical microscope. $20 \mathrm{~mm}$ cylindrical rods were cut from each sample, grounded, polished and etched in Nital prior to microstructural analysis. The microstructure was taken with magnification of 500 .

\section{Hardness test}

Prior to testing, specimens were mounted using phenolic powder, grinded and polished to obtain a smooth surface. A direct load of 
Table 2. Mechanical properties of quenched low Carbon steel at different immersion speeds.

\begin{tabular}{ccccccc}
\hline $\begin{array}{c}\text { Immersion speed } \\
(\mathbf{m} / \mathbf{s})\end{array}$ & Tensile strength $\mathbf{( N / \mathbf { m m } ^ { 2 } )}$ & \% elongation & Modulus of elasticity & $\begin{array}{c}\text { Yield strength } \\
\left(\mathbf{N} / \mathbf{m m}^{2}\right)\end{array}$ & $\begin{array}{c}\text { Hardness } \\
(\text { Case })\end{array}$ & $\begin{array}{c}\text { Hardness } \\
(\text { Core })\end{array}$ \\
\hline 0.3102 & 82.18 & 55.8 & 5674.3 & 312.1 & 166.25 & 162.55 \\
0.3241 & 71.05 & 53.1 & 8836.9 & 298.7 & 158.35 & 132 \\
0.3384 & 81.68 & 46.5 & 4642.9 & 215.4 & 136.3 & 110.95 \\
0.4167 & 70.39 & 42.6 & 10576.8 & 241.2 & 157.85 & 115.3 \\
\hline
\end{tabular}

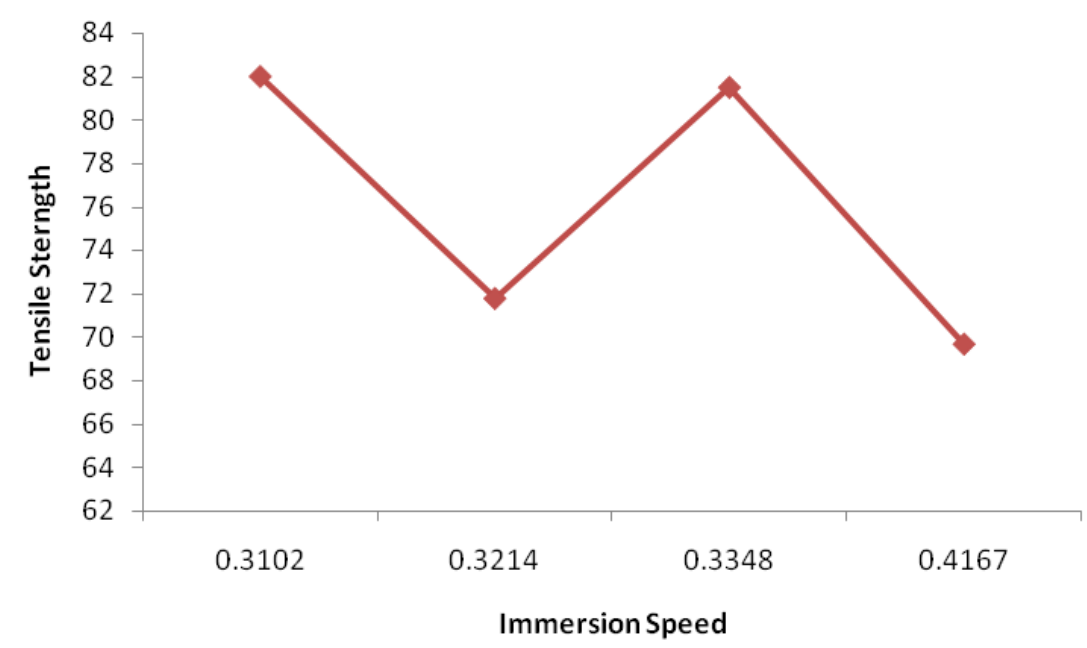

Figure 2. Tensile strength $\left(\mathrm{N} / \mathrm{mm}^{2}\right)$ against Immersion speed $(\mathrm{m} / \mathrm{s})$.

$490 \mathrm{~N}$ was applied for a dwell time of $10 \mathrm{~s}$ and hardness readings were evaluated using Leitz 8299 micro-hardness tester. The indentation was square shaped. Diagonal length of square was measured by scale attached on the microscope. Hardness was obtained directly from the chart given in the manual for corresponding load and diagonal length. Multiple hardness tests were performed on each sample and the average of the values was taken as a measure of the hardness value for each specimen.

\section{Tensile test}

P2000 electronic tensiometer was used to conduct the test following standard test procedures in accordance with the ASTM E8M-91 standards (1992). The samples were tested at a nominal strain rate of $10^{-3} / \mathrm{s}$ until fracture. The tensile properties evaluated from the engineering stress-strain curves developed from the tension test are - the ultimate tensile strength $\left(\sigma_{\mathrm{u}}\right)$, the yield strength $\left(\sigma_{y}\right)$, and the strain to fracture $\left(\varepsilon_{f}\right)$.

\section{RESULTS AND DISCUSSION}

\section{Effect of heat treatment on mechanical properties}

The effect of immersion speed on the mechanical properties (ultimate tensile strength, hardness, toughness, percentage elongation, and percentage reduction) of the quenched steel is shown in the Table 2.

\section{Effect on tensile strength}

As the immersion speed of the quenched steel increases, the tensile strength of the samples fluctuates, resulting in variation between the tensile strength and speed of immersion. In Figure 2, it was observed that at low immersion speed of $0.3102 \mathrm{~m} / \mathrm{s}$, maximum tensile strength of $82.18 \mathrm{~N} / \mathrm{mm}^{2}$ was obtained. At the highest immersion speed of $0.4167 \mathrm{~m} / \mathrm{s}$, the tensile strength reduces to $70.39 \mathrm{~N} / \mathrm{mm}^{2}$ which shows no stable relationship between the immersion speed and the tensile strength.

\section{Effect on percentage elongation}

Figure 3 shows the relationship between the percentage elongation and immersion speed. As immersion speed 


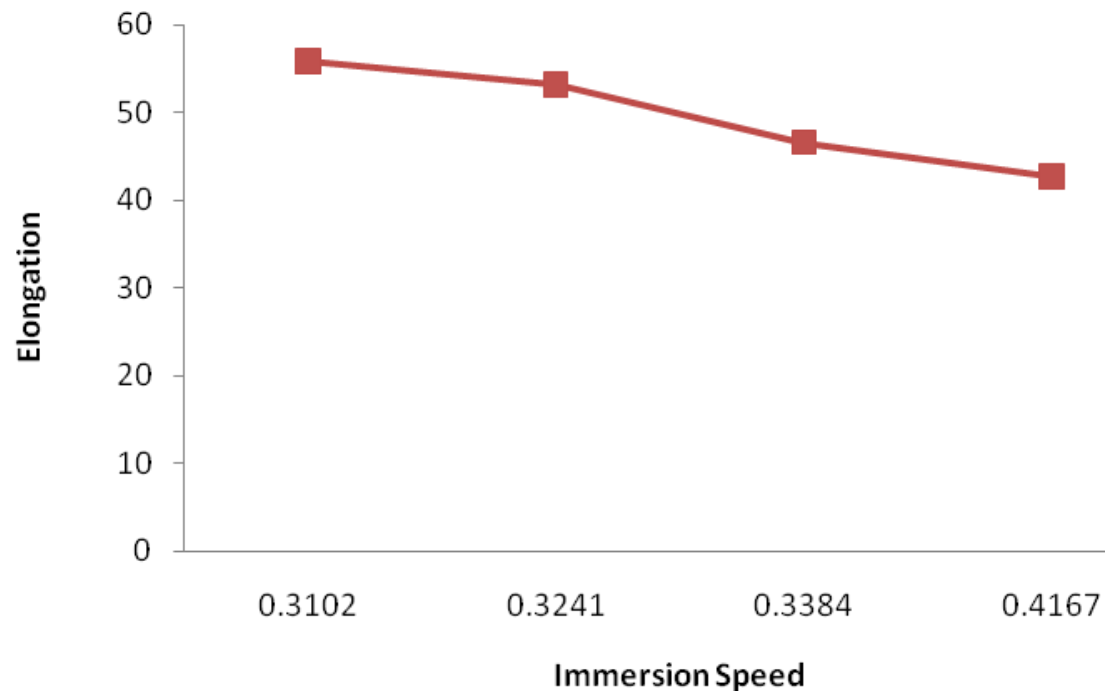

Figure 3. Percentage elongation against immersion speed (m/s).

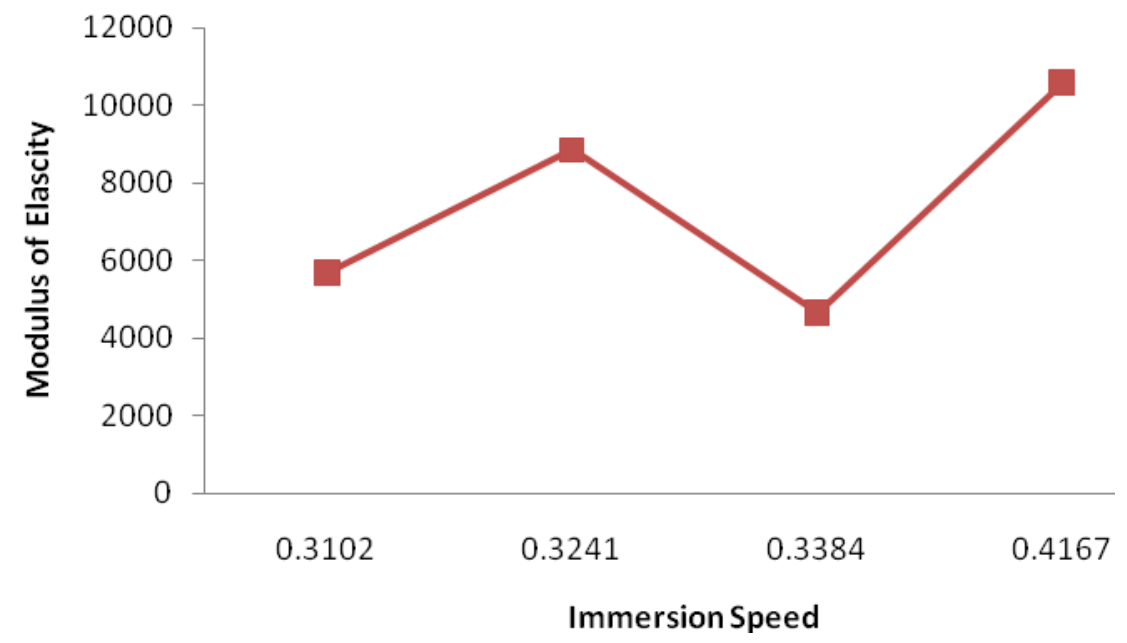

Figure 4. Modulus of elasticity $\left(\mathrm{N} / \mathrm{mm}^{2}\right)$ against Immersion speed $(\mathrm{m} / \mathrm{s})$.

increases, the percentage elongation of the specimens decreases. This shows an inverse relationship between the percentage elongation and immersion speed. At a low immersion speed of $0.3102 \mathrm{~m} / \mathrm{s}$, high percentage elongation of $55.15 \%$ was obtained above which the percentage elongation of the samples decreases.

\section{Effect on elasticity}

The modulus elasticity of the quenched mild steel samples also fluctuates in relation with increased immersion speed. From Figure 4, the modulus of elasticity increases from 5674.3 to 8836.9 as the immersion speed increases from 0.3102 to $0.3214 \mathrm{~m} / \mathrm{s}$. For the next immersion speed of $0.3384 \mathrm{~m} / \mathrm{s}$, the modulus of elasticity drops drastically to 4642.9 and increases to 10576.8 for the increased immersion speed of $0.4167 \mathrm{~m} / \mathrm{s}$.

\section{Effect on yield strength}

Figure 5 shows the relationship between the yield 


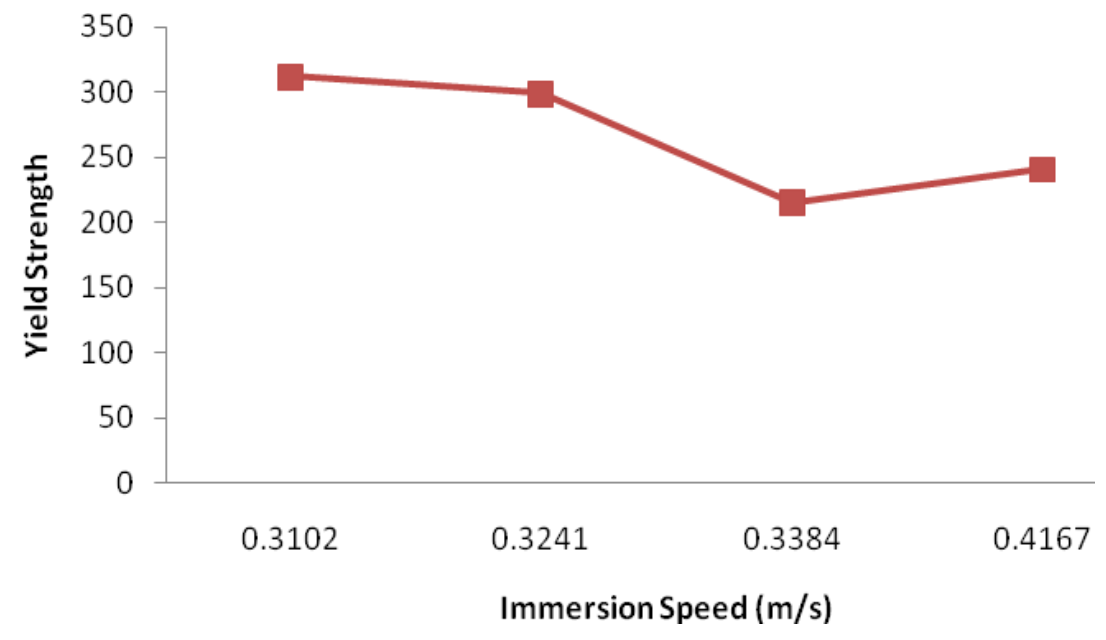

Figure 5. Yield Strength $\left(\mathrm{N} / \mathrm{mm}^{2}\right)$ against Immersion speed $(\mathrm{m} / \mathrm{s})$.

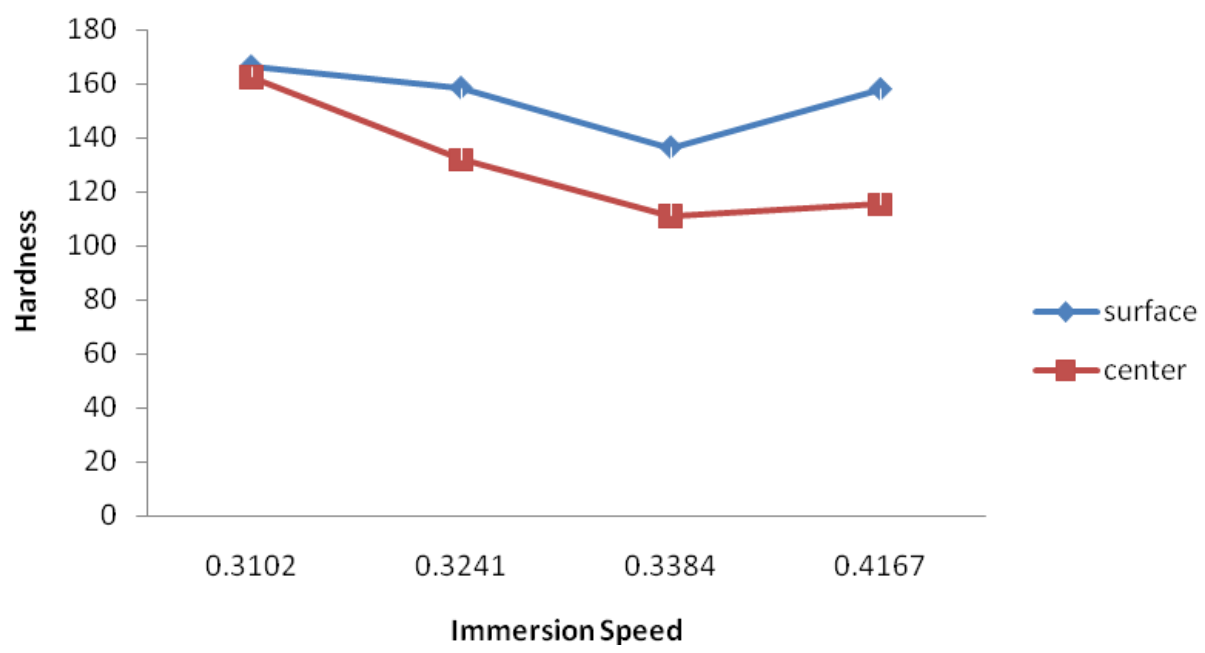

Figure 6. Hardness value (BHN) against immersion speed $(\mathrm{m} / \mathrm{s})$ (surface and center).

strength and immersion speed. The yield strength of the steel samples reduces with increase in immersion rate from 0.3102 to $0.3384 \mathrm{~m} / \mathrm{s}$. Optimum yield strength of 310 was obtained at low immersion speed of $0.3102 \mathrm{~m} / \mathrm{s}$.

\section{Effect on hardness}

There is a notable difference between the hardness of the steel samples at the surface and at the center (Figure 6). Although, the hardness of the specimens had an inverse relation with the immersion speed, i.e. the higher the speed of immersion, the lower the hardness value at both the surface and the center. It was evidence that the hardness values at the surface of the samples are higher than that at the center. This can be attributed to the fact that quenching occurs faster and more rapidly at the surface than at the center.

\section{Effect of immersion speed on the microstructure of the steel}

The micrographs of the steel samples quenched at different immersion speed were shown in Figures 7 to 10 . The massive martensite structure of hardened sample, 


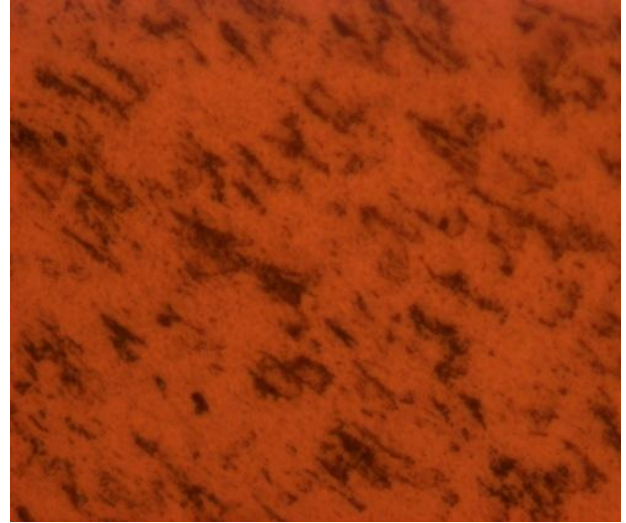

Surface

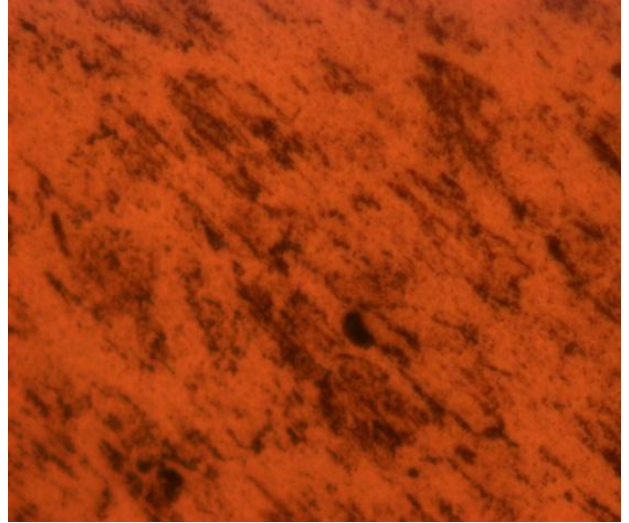

Core

Figure 7. Microstructure of oil quenched steel at immersion rate of $0.3102 \mathrm{~m} / \mathrm{s}$.

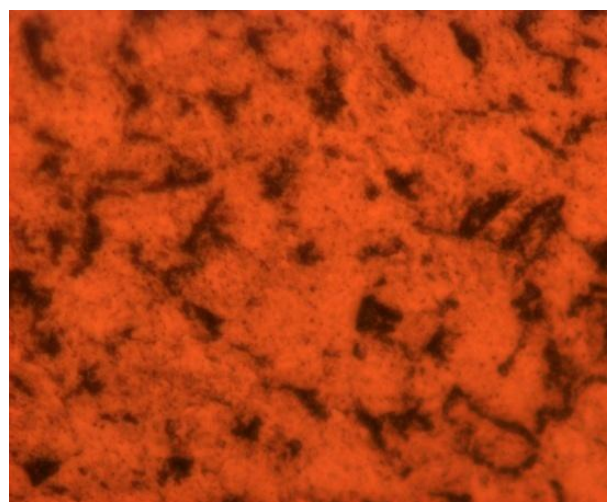

Surface

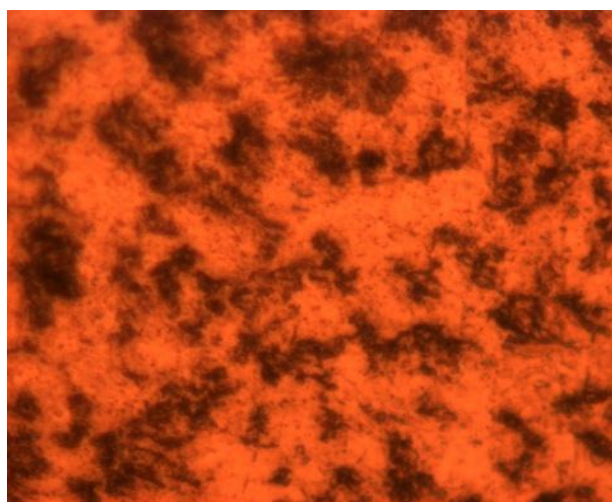

Core

Figure 8. Microstructure of oil quenched steel at immersion rate of $0.3241 \mathrm{~m} / \mathrm{s}$.

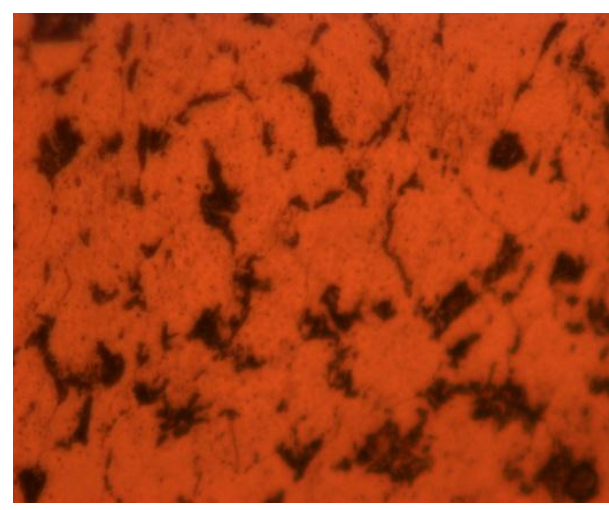

Surface

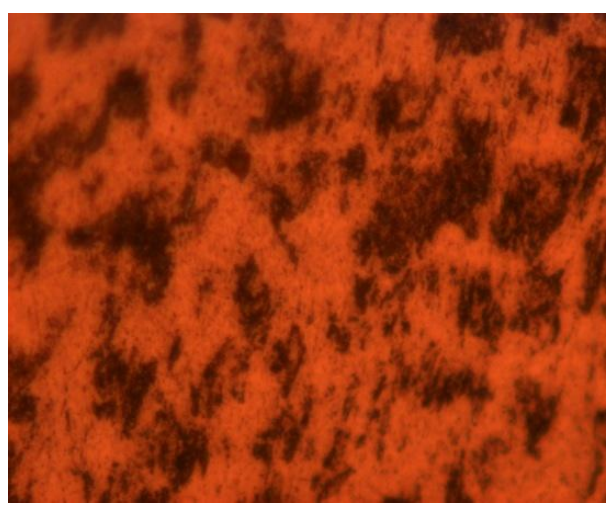

Core

Figure 9. Microstructure of oil quenched steel at immersion rate of $0.3384 \mathrm{~m} / \mathrm{s}$. 


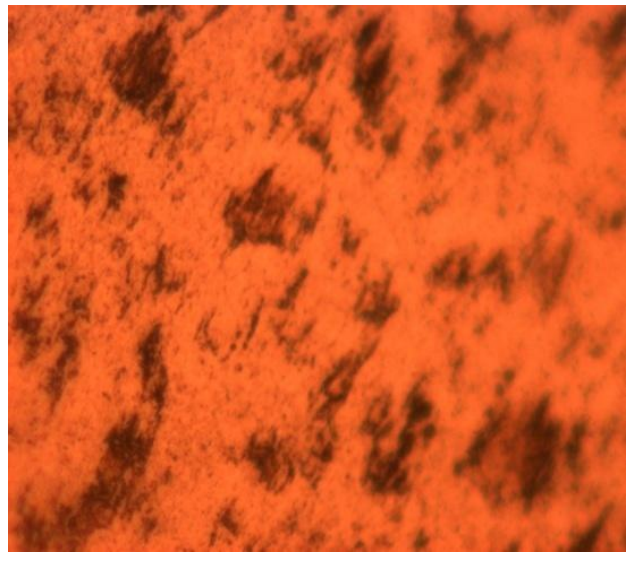

Surface

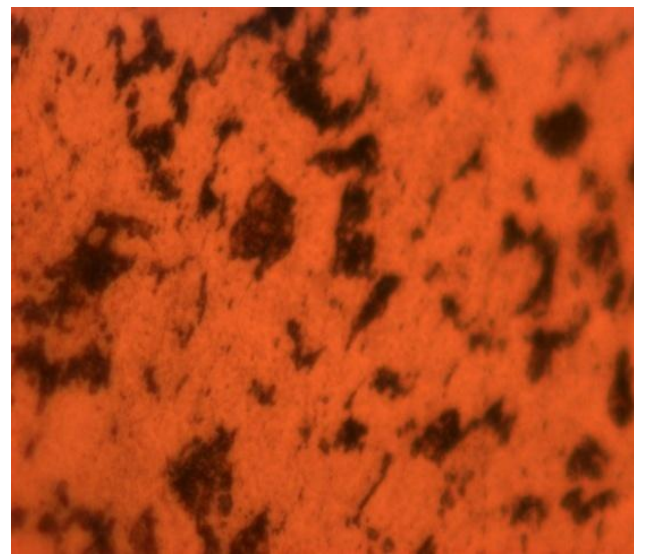

Core

Figure 10. Microstructure of oil quenched steel at immersion rate of $0.4167 \mathrm{~m} / \mathrm{s}$.

when low Carbon steels are rapidly quenched from its austenite temperature to room temperature, the austenite will decompose into a mixture of some medium Carbon martensite and fewer pearlite as a result increases the tensile strength, hardness and little reduction in ductility of the material. In this study, it was observed that the surface (outer layer) of the steel samples cooled more rapidly resulting in large martensitic formation. Little pearlite were noticed at the surface compared to the center (inner layer). This varied effect may be due to the fact that the surface has contact with the oil quenchant; hence, cools faster than the center. The high martensitic composition may make the material very hard and brittle. As the speed of immersion decreases, the martensitic formation also decreases, giving room for more pearlite formation, hence decrease in the hardness as the speed of immersion increases. Obviously, the rates of immersion bring about a variation in the hardness of the material.

\section{Conclusions}

The speeds of immersion have varying effects on individual mechanical properties of AISI 1020. Mechanical properties such as yield strength and percentage elongation decreases while young modulus increases as the material is quenched. The tensile strength of the mild steel samples varied as the immersion speeds. The effect of immersion speeds on the hardness of the steel differs at the center and at the core. As the immersion speed increases, the hardness at the surface increases as compared to the hardness value at the center of the mild steel samples.

\section{Conflict of Interest}

The authors have not declared any conflict of interest.

\section{REFERENCES}

Çalik A (2009). Effect of cooling rate on hardness and microstructure of AISI 1020, AISI 1040 and AISI 1060 Steels. Int. J. Phys. Sci. 4(9):514-518.

Fadare DA, Fadara TG, Akanbi OY (2011). Effect of Heat Treatment on Mechanical Properties and Microstructure of NST 37-2 Steel." J. Min. Mater. Character. Eng. 10(3):299-308.

Odusote JK, Ajiboye TK, Rabiu AB (2012). Evaluation of Mechanical Properties of Medium Carbon Steel Quenched in water and Oil. J. Technol. 15(4):218-224.

Mackerle $\mathrm{J}$ (2003). Finite element analysis and simulation of quenching and other heat treatment processes. A bibliography (1976-2001): Comput. Mater. Sci. 27:313-332.

Sharma JP, Chaturvedi S, Arora P (2012). Studies on effect of percentage of Carbon on the tensile and compressive strength of structural steel. Int. J. Eng. Sci. Technol. 4:5. 